\title{
The Metabolic Syndrome and Sexual Dysfunction in a State of Inflammation
}

\author{
Huseini Alidu', W. K. B. A. Owiredu², Nafiu Amidu³, Peter Paul Mwinsanga Dapare3, \\ Ahmed Tijani Bawah1, Christian Kofi Gyasi-Sarpong", Christian Obirikorang²

\begin{abstract}
${ }^{1}$ Department of Medical Laboratory Science, University of Health and Allied Sciences, Ho, Ghana
${ }^{2}$ Department of Molecular Medicine, Kwame Nkrumah University of Science \& Technology, Kumasi, Ghana

${ }^{3}$ Department of Biomedical Laboratory Science, University for Development Studies, Tamale, Ghana

${ }^{4}$ Department of Surgery (Urology Unit), Komfo Anokye Teaching Hospital, Kumasi, Ghana

Email: wiisibie@yahoo.com,wkbaowiredu@yahoo.com,nafamidu@yahoo.com, peterpauldapare@yahoo.com,
\end{abstract} \\ ahmed024gh@yahoo.com,gaysek@yahoo.com,krisobiri@yahoo.com
}

How to cite this paper: Alidu, H., Owiredu, W.K.B.A., Amidu, N., Dapare, P.P.M., Bawah, A.T., Gyasi-Sarpong, C.K. and Obirikorang, C. (2017) The Metabolic Syndrome and Sexual Dysfunction in a State of Inflammation. Advances in Sexual Medicine, 7, 82-96.

https://doi.org/10.4236/asm.2017.72006

Received: January 30, 2017

Accepted: April 7, 2017

Published: April 10, 2017

Copyright $\odot 2017$ by authors and Scientific Research Publishing Inc. This work is licensed under the Creative Commons Attribution International License (CC BY 4.0).

http://creativecommons.org/licenses/by/4.0/

\begin{abstract}
Background: Diabetes is amongst the most researched diseases worldwide due to its debilitating effects on patients. Diabetics have a higher risk of developing erectile dysfunction. Evidence has linked erectile dysfunction severity to increased age, duration of diabetes, the metabolic syndrome and hypogonadism. However, the role of inflammatory markers in the pathogenesis of $\mathrm{SD}$ is still unclear. The contributions of hormonal parameters and adipokines to both macro and micro vascular complications are still poorly understood. This study was conducted to provide some evidence of an association between SD, the metabolic syndrome and inflammatory hormones. Method: A total of 130 diabetic males participated in this study. All participants were at least 18 years and were actively engaged in a stable heterosexual relationship for a minimum of two years before they were recruited for this study. The Diabetic men were evaluated using the Golombok Rust Inventory of Sexual Satisfaction for males (GRISS-M). The NCEP-ATP III, IDF and WHO criteria were used to assess the metabolic syndrome. Blood samples were taken for biochemical and hormonal assays. Results: Participants with raised Triglyceride levels showed significantly higher leptin levels for NCEP-ATP III and IDF criteria. Participants with impotence showed significantly lower leptin/adiponectin ratio $(\mathrm{p}=0.0184)$ whilst participant with non-sensuality showed significantly higher values of leptin/adiponectin ratio $(p=0.0103)$. A significantly higher levels of insulin $(p=0.0265)$ was recorded in participants with Dissatisfaction problems. Participants with Non communication problems showed significantly higher levels of insulin $(\mathrm{p}=0.0419)$ and lower levels of adiponectin ( $\mathrm{p}$ $=0.0438$ ) whilst participants with infrequency showed significantly lower levels of adiponectin $(\mathrm{p}=0.0042)$. Conclusion: The relative balance between
\end{abstract}


pro inflammatory and anti-inflammatory mediators of endothelial dysfunction could possibly be essential for the progression of diabetes to SD and the MetS thus a longitudinal study which measures a range of both pro and anti-inflammatory hormones and adipokines as risk factors for SD and the MetS could potentially aid in improvements in assessing the risk of developing these conditions among diabetics.

\section{Keywords}

Golombuk-Rust Inventory for Sexual Satisfaction-Male, Metabolic Syndrome, Sexual Dysfunction

\section{Introduction}

Diabetes is one of the most researched diseases in the world due to its debilitating effects on patients and the many complications that could result from this disease. The number of people with diabetes is known to be increasing due to population growth, aging, urbanization and increasing prevalence of obesity and physical inactivity [1]. Diabetics are reported to have a higher risk of developing erectile dysfunction (ED) [2] [3] [4], sexual dysfunction (SD) [5] [6] and other cardiovascular pathologies [6] [7]. Evidence has linked erectile dysfunction severity to increased age [8] and duration of diabetes [8], poor glycaemic control and cardiovascular disease [8] as well as the metabolic syndrome [9]. Chronic hyperglycaemias as well as dyslipidaemia underpinned by endothelial dysfunction are the major underlying biochemical factors in diabetes [10]. The contribution of these abnormalities to both macro and micro vascular complications is well known, however their contribution to SD or specific domains of SD and which specific pathophysiological mechanism are implicated is still open to debate. Furthermore, the pathophysiological role of inflammatory hormones and their roles in the development or worsening of SD and the MetS is still not clearly understood.

The metabolic syndrome (MetS) is also characterized by a cluster of factors such as hyperglycemia, dyslipidaemia, high blood pressure, obesity and insulin resistance and these have long been implicated in the causation or worsening of diabetes [11]. Similarities in aetiology of the MetS with diabetes as well as SD can be traced to endothelial dysfunction and it is therefore not surprising that an increased prevalence of SD and MetS is largely reported to be among diabetics [9]. Although the pathophysiology is incompletely understood, insulin resistance and abdominal obesity are central to subsequent abnormalities in circulating glucose and lipoproteins and vascular function that lead to type 2 diabetes, atherosclerosis and CVD [12]. Various researches have attempted to elucidate the relationship between SD, MetS, inflammatory markers and some hormonal parameters which are likely to influence this relationship with some evidence showing a positive association between fasting insulin levels and inverse relationship between free testosterone levels, SHBG and the development of diabetes [13]. 
Some research has shown that age related decrease in testosterone levels is significantly exacerbated in obese men with the metabolic syndrome [14] but the role of inflammatory hormones is yet to be fully elucidated. It is in this light that this research seeks to provide some evidence of an association between MetS, SD and some hormonal parameters which are potential markers of inflammation. This research therefore seeks to determine if there are differences in the measured hormonal parameters between subjects with and without SD and/or the MetS.

Adipocytes play an important role in regulating triglycerides and free fatty acids levels [15]. High levels of androgens drive differentiation towards myogenesis and thus inhibit adipogenesis [16]. Thus androgen levels as well as levels of hormones produced by these adipocytes are likely to influence the development of the MetS in both sexes. A plausible biological mechanism for obesity induced hypogonadism may result in part from increased feedback inhibition of the hypothalamic-pituitary axis due to high levels of estrogen in obese men [17].

The Third National Health and Nutrition Examination Survey [17] reported that low free and bioavailable testosterone concentrations in the normal range were associated with diabetes, independent of adiposity. They suggested that low androgen levels may be a risk factor for diabetes in men. In a multivariable model adjusted for age, ethnicity and adiposity they observed that men in the first tertile (lowest) of free testosterone level were four times more likely to have prevalent diabetes compared with men in the third tertile. Similarly, men in the first tertile of bioavailable testosterone also were approximately four times as likely to have prevalent diabetes compared with men in the third tertile, these associations persisted even after excluding men with clinically abnormal testosterone concentrations. Others researchers [18] have demonstrated that the relationship between testosterone levels and adiposity is even dose dependent, with the effect of a $5 \mathrm{mg}$ testosterone patch on body fat reduction being less in comparison to a $100 \mathrm{mg}$ testosterone patch administered over the course of three months. They also showed that treatment with testosterone gel improved sexual function and mood, lean and fat mass as well as bone mineral density in hypogonadal men. Whether these established effects of testosterone on sexual function and body adiposity markers is mediated or influenced by inflammatory markers is still open to debate.

\section{Methods}

A total of 130 diabetic males who were attending the diabetic clinic and managed on oral diabetic medications at the Maamobi General Hospital in Accrabetween October, 2013 to September, 2014 were recruited for this study. Participants were consecutively sampled and those who were at least 18 years and were actively engaged in a stable heterosexual relationship were enrolled in the study. A stable heterosexual relationship was defined as having engaged in a continuous heterosexual relationship for a minimum of two years. An informed and signed consent was obtained from the participants before enrollment into the 
study. Ethical approval was obtained from the Committee on Human Research Publication and Ethics (CHRPE) of the School of Medical Science and the Komfo Anokye Teaching Hospital (KATH), Kumasi. The Diabetic men were evaluated blindly using the Golombok Rust Inventory of Sexual Satisfaction for males (GRISS-M) and they were further categorized into the various subscales of SD. The NCEP-ATP III, IDF and WHO criteria of the MetS were used to assess the metabolic syndrome and the participants were categorized into the various MetS components as well as MetS scores. Eight (8) mls of blood was obtained for biochemical and hormonal analysis. Fasting blood samples were collected from each participant in the morning between 06.00 to 09.00 GMT into fluoride oxalate tube and evacuated gel tubes for serum preparation (Becton Dickinson, $\mathrm{Ru}$ therford, NJ). Samples in the fluoride oxalate tubes were used for fasting blood glucose measurement after centrifugation at $18^{\circ} \mathrm{C}$ whilst samples in the evacuated gel tubes were centrifuged at $3000 \mathrm{~g}$ for 5 minutes at $18^{\circ} \mathrm{C}$ and the serum aliquoted and stored in cryovials at a temperature of $-80^{\circ} \mathrm{C}$ until time for biochemical and hormonal assay. Lipid profile and fasting blood glucose levels were determined using the BT $5000^{\circ}$ Random Access Chemistry Analyzer (Biotecnica, Italy), the JAS Diagnostics ${ }^{\oplus}$ reagent kits were used in all of these assays. The hormonal assays were done using the Elabscience ${ }^{\circledast}$ reagent kits on the AxSYManalyser. The Sandwich-ELISA method was used in measuring the levels of leptin and adiponectin whilst the competitive ELISA method was used to assay Insulin levels. The methods adopted by the automated instruments for the determination of biochemical and hormonal parameters were done according to the reagent manufacturers' instructions (JAS Diagnostics, Inc. Miami Florida, USA and Elabscience Biotechnology Co. Ltd, Hubei Province, China). The Metabolic syndrome was defined by the NCEP-ATP III to include individuals with any three or more of the following five components: 1) abdominal obesity (waist circumference $>102 \mathrm{~cm}$ ); 2) high TG $\geq 1.7 \mathrm{mmol} / \mathrm{L} ; 3$ ) low HDL-C: $<0.9 \mathrm{mmol} / \mathrm{L}$; and 4) High BP (systolic BP $\geq 130 \mathrm{~mm} \mathrm{Hg}$ or diastolic BP $\geq 85 \mathrm{~mm} \mathrm{Hg}$ or treatment of hypertension); and 5) high fasting glucose $\geq 6.1 \mathrm{mmol} / \mathrm{l}$ (NCEP, 2001). The IDF criteria of the MetS was diagnosed if central obesity (waist measurement $>90$ $\mathrm{cm}$ ) was accompanied by any 2 of the following 4 factors: 1) TG levels of 1.7 $\mathrm{mmol} / \mathrm{L}$ or greater, 2) an HDL cholesterol lower than $1.03 \mathrm{mmol} / \mathrm{L}, 3)$ a blood pressure (BP) of 130/85 mm Hg or greater or treatment of previously diagnosed hypertension, and 4) a fasting blood glucose (FBG) of $5.6 \mathrm{mmol} / \mathrm{L}$ or greater or previously diagnosed type 2 diabetes. The WHO criteria was definedby the presence of diabetes mellitus, impaired glucose tolerance and any two of the following: 1) Body mass index (BMI) $\geq 30 \mathrm{~kg} / \mathrm{m}^{2}$ and/or waist-to-hip ratio $>0.90,2$ ) blood pressure $\geq 140 / \geq 90 \mathrm{mmHg}$ or on medication, 3) diabetes $\geq 6.1 \mathrm{mmol} / \mathrm{L}$ or on medication for diabetes, impaired glucose tolerance, 4) triglyceride $\geq 1.7$ $\mathrm{mmol} / \mathrm{L}$ and/or HDL-C $<0.91 \mathrm{mmol} / \mathrm{L}$.

\section{Statistical Analysis}

All data analysis were performed using the SPSS software, version 11.0 systat, 
Inc. Germany and GraphPad Prism, version 5.0, San Diego California, USA. Data presentation was done as the mean \pm SD or percentages. In all the statistical analysis, a value of $\mathrm{p}<0.05$ was considered to be significant.

\section{Results}

The age range for those who responded was between 29 to 89 years, with a mean age of $63.04 \pm 10.85$ years and a mean duration of diabetes of $8.38 \pm 6.53$ years. The mean weight and BMI of the respondents was $82.20 \pm 30.48 \mathrm{~kg}$ and $28.88 \pm$ $11.32 \mathrm{~kg} / \mathrm{m}^{2}$ respectively. The mean SBP and DBP recorded was $157.50 \pm 25.27$ $\mathrm{mmHg}$ and $101.60 \pm 14.96 \mathrm{mmHg}$ respectively. The mean WC, HC and WHR were $95.25 \pm 10.12 \mathrm{~cm}, 101.40 \pm 8.70 \mathrm{~cm}$ and $0.94 \pm 0.04$ respectively. When the participants were stratified by sexual function, participants with SD were significantly older (66.67 \pm 11.8 against $61.05 \pm 9.79)$, and had a longer duration of diabetes $(10.00 \pm 7.79$ against $7.53 \pm 5.62)$ but there were no significant differences in the SBP, DBP, weight, BMI, waist circumference, hip circumference and waist to hip ratio amongst participants with or without SD (Table 1).

Table 1. General characteristics of the study population.

\begin{tabular}{|c|c|c|c|c|}
\hline Variable & $\begin{array}{c}\text { Total } \\
(n=130)\end{array}$ & $\begin{array}{l}\text { NO SD } \\
(n=46)\end{array}$ & $\begin{array}{c}S D \\
(n=84)\end{array}$ & P Value \\
\hline Age (years) & $63.04 \pm 10.85$ & $60.98 \pm 9.80$ & $66.67 \pm 11.84$ & 0.0043 \\
\hline Duration of diabetes (years) & $8.38 \pm 6.53$ & $7.53 \pm 5.62$ & $10.00 \pm 7.79$ & 0.0418 \\
\hline \multicolumn{5}{|c|}{ Anthropometric Data } \\
\hline $\mathrm{SBP}(\mathrm{mmHg})$ & $157.50 \pm 25.27$ & $157.60 \pm 27.86$ & $157.50 \pm 24.09$ & 0.9841 \\
\hline $\mathrm{DBP}(\mathrm{mmHg})$ & $101.60 \pm 14.96$ & $98.74 \pm 14.13$ & $103.20 \pm 15.34$ & 0.2572 \\
\hline Weight (kg) & $82.20 \pm 30.48$ & $85.79 \pm 32.38$ & $80.24 \pm 29.60$ & 0.4869 \\
\hline Height (m) & $1.69 \pm 0.07$ & $1.69 \pm 0.07$ & $1.69 \pm 0.07$ & 0.9317 \\
\hline BMI $\left(\mathrm{kg} / \mathrm{m}^{2}\right)$ & $28.88 \pm 11.32$ & $28.27 \pm 11.32$ & $29.99 \pm 11.37$ & 0.4116 \\
\hline $\mathrm{HC}(\mathrm{cm})$ & $101.40 \pm 8.70$ & $101.10 \pm 8.02$ & $101.50 \pm 9.14$ & 0.8523 \\
\hline $\mathrm{WC}(\mathrm{cm})$ & $95.25 \pm 10.12$ & $96.20 \pm 9.60$ & $94.73 \pm 10.47$ & 0.5796 \\
\hline WHR & $0.94 \pm 0.04$ & $0.95 \pm 0.04$ & $0.93 \pm 0.04$ & 0.0970 \\
\hline \multicolumn{5}{|c|}{ Biochemical parameters } \\
\hline FBG $(\mathrm{mmol} / \mathrm{L})$ & $8.73 \pm 2.82$ & $7.97 \pm 2.65$ & $9.15 \pm 2.85$ & 0.1076 \\
\hline Total cholesterol (mmol/L) & $4.52 \pm 1.31$ & $4.78 \pm 1.42$ & $4.37 \pm 1.24$ & 0.2264 \\
\hline Triglyceride (mmol/L) & $0.92 \pm 0.49$ & $0.89 \pm 0.42$ & $0.94 \pm 0.52$ & 0.6917 \\
\hline HDL-cholesterol (mmol/L) & $1.39 \pm 0.51$ & $1.53 \pm 0.60$ & $1.31 \pm 0.45$ & 0.0992 \\
\hline LDL-cholesterol (mmol/L) & $2.67 \pm 0.97$ & $2.78 \pm 1.11$ & $2.60 \pm 0.89$ & 0.4709 \\
\hline \multicolumn{5}{|c|}{ Sexual Dysfunction } \\
\hline Impotence & $11.95 \pm 1.54$ & $11.09 \pm 0.78$ & $12.43 \pm 1.65$ & $<0.0001$ \\
\hline Premature Ejaculation & $8.69 \pm 1.86$ & $7.78 \pm 1.49$ & $9.19 \pm 1.85$ & $<0.0001$ \\
\hline Non Sensuality & $11.31 \pm 1.31$ & $10.65 \pm 0.92$ & $11.67 \pm 1.35$ & $<0.0001$ \\
\hline Avoidance & $10.66 \pm 2.22$ & $11.26 \pm 2.13$ & $10.33 \pm 2.20$ & 0.0219 \\
\hline Dissatisfaction & $10.89 \pm 1.04$ & $10.22 \pm 1.03$ & $11.26 \pm 0.85$ & $<0.0001$ \\
\hline Non Communication & $5.15 \pm 0.79$ & $4.78 \pm 0.79$ & $5.36 \pm 0.72$ & $<0.0001$ \\
\hline Infrequency & $5.86 \pm 0.79$ & $5.65 \pm 0.48$ & $5.98 \pm 0.89$ & 0.0239 \\
\hline
\end{tabular}


The mean FBG, total cholesterol, triglyceride, HDL-cholesterol and LDLcholesterol levels for the participants were $8.73 \pm 2.82 \mathrm{mmol} / \mathrm{L}, 4.52 \pm 1.31$ $\mathrm{mmol} / \mathrm{L}, 0.92 \pm 0.49 \mathrm{mmol} / \mathrm{L}, 1.39 \pm 0.51 \mathrm{mmol} / \mathrm{L}$ and $2.67 \pm 0.97 \mathrm{mmol} / \mathrm{L}$ respectively. When the participants were stratified by sexual function, participants with SD recorded no significant difference in their biochemical parameters in comparism with those without SD (Table 1).

The SD scores recorded for impotence, PE, nonsensuality, avoidance, dissatisfaction, non communication and infrequency were $4.99 \pm 2.05,4.66 \pm 1.77$, $5.15 \pm 2.05,4.86 \pm 1.94,4.82 \pm 2.02,5.12 \pm 1.88$ and $4.72 \pm 1.51$. Participants with SD recorded significantly higher scores for impotence $(5.60 \pm 2.19$ against $3.87 \pm 1.14), \mathrm{PE}(5.14 \pm 1.73$ against $3.78 \pm 1.51)$, nonsensuality $(5.70 \pm 2.07$ against $4.17 \pm 1.64)$, dissatisfaction $(5.52 \pm 1.71$ against $3.52 \pm 1.93)$ as well as non communication (5.60 \pm 1.70 against $4.26 \pm 1.94)$ (Table 1$)$.

The mean insulin, adiponectin, leptin and the leptin/adiponectin ratio recorded among the participants was $83.10 \pm 22.79 \mathrm{pg} / \mathrm{ml}, 1.51 \pm 0.35 \mathrm{ng} / \mathrm{ml}, 24.23$ $\pm 12.00 \mathrm{ng} / \mathrm{ml}$ and $0.02 \pm 0.00$ respectively (Table 2 ). When the study participants were stratified by the MetS using the various criteria, the NCEP-ATP III recorded no significant differences in the insulin, leptin and the leptin/adiponectin ratio between subjects with or without the MetS. However participants with the MetS recorded increased adiponectin levels. The IDF and WHO criteria however did not show any significant difference in any of these parameters amongst subjects with or without the MetS (Table 2). When the mean values of

Table 2. Hormonal assays stratified by Presence or Absence of MetS.

\begin{tabular}{|c|c|c|c|}
\hline VariableTotal & No MetS & MetS & P Value \\
\hline \multicolumn{4}{|c|}{ ATP } \\
\hline $\begin{array}{c}\text { Insulin }(\mathrm{pg} / \mathrm{ml}) \\
83.10 \pm 22.79\end{array}$ & $104.22 \pm 64.46$ & $37.65 \pm 21.30$ & 0.1354 \\
\hline $\begin{array}{c}\text { Adiponectin }(\mathrm{ng} / \mathrm{ml}) \\
1.51 \pm 0.35\end{array}$ & $1.31 \pm 0.43$ & $1.81 \pm 0.32$ & 0.0450 \\
\hline $\begin{array}{c}\text { Leptin }(\mathrm{ng} / \mathrm{ml}) \\
24.23 \pm 12.00\end{array}$ & $22.40 \pm 10.12$ & $27.43 \pm 13.35$ & 0.2556 \\
\hline $\begin{array}{l}\text { Leptin/Adiponectin Ratio } \\
\qquad 0.02 \pm 0.00\end{array}$ & $0.02 \pm 0.00$ & $0.01 \pm 0.00$ & 0.4764 \\
\hline \multicolumn{4}{|c|}{ IDF } \\
\hline Insulin (pg/ml) & $69.54 \pm 37.36$ & $97.76 \pm 48.33$ & 0.2689 \\
\hline Adiponectin (ng/ml) & $1.58 \pm 0.40$ & $1.42 \pm 0.50$ & 0.4710 \\
\hline Leptin (ng/ml) & $23.43 \pm 13.31$ & $24.47 \pm 10.43$ & 0.2590 \\
\hline Leptin/Adiponectin Ratio & $0.01 \pm 0.0$ & $0.02 \pm 0.00$ & 0.0725 \\
\hline \multicolumn{4}{|c|}{ WHO } \\
\hline Insulin (pg/ml) & $42.97 \pm 32.65$ & $102.43 \pm 75.82$ & 0.0922 \\
\hline Adiponectin (ng/ml) & $1.74 \pm 0.30$ & $1.39 \pm 0.80$ & 0.1033 \\
\hline Leptin (ng/ml) & $23.36 \pm 13.46$ & $45.15 \pm 22.53$ & 0.3256 \\
\hline Leptin/Adiponectin Ratio & $0.01 \pm 0.00$ & $0.03 \pm 0.01$ & 0.2753 \\
\hline
\end{tabular}


the biochemical parameters for the diabetics who recorded $0,1,2,3$ and 4 component scores of the MetS were compared for intergroup variations using the ATP III and IDF classifications, there were no significant differences recorded for insulin, adiponectin, leptin and leptin/adiponectin ratio but the WHO criteria recorded significant differences in the component score range with a generally increasing trend of adiponectin from 1 to 4 component scores (Table 3 ).

As shown in Table 4 and Table 5, when the diabetic participants were stratified based on the presence or absence of the MetS components with regards to the various hormonal parameters, participants with the presence of Abdominal Obesity, represented by WC, showed no significant difference in the levels of insulin, adiponectin, leptin and Leptin/Adiponectin ratios for the NCEP-ATP III and IDF MetS criteria. This observation was also recorded for diabetic participants who recorded the presence of Central obesity as represented by WHR using the WHO MetS criteria.

Diabetic male participants with raised FBG showed significantly higher levels of insulin for NCEP-ATP III (104.63 \pm 76.39 against 29.44 \pm 13.80$)$, IDF (106.45 \pm 79.74 against $42.63 \pm 12.80)$ and the WHO criteria $(97.48 \pm 48.74$ against 42.33 \pm 28.76 ) in comparison with participants without raised FBG. Participants with raised Triglyceride levels showed significantly higher leptin levels for NCEPATP III (46.44 \pm 22.24 against $21.32 \pm 10.65)$ and IDF criteria (44.27 \pm 24.92 against $20.26 \pm 11.27)$. There was however no observed differences between subjects with or without the presence of raised BP in their levels of insulin, adiponectin, leptin and Leptin/Adiponectin ratios irrespective of the MetS criteria used.

Table 3. Hormonal parameters stratified by MetS component scores.

\begin{tabular}{|c|c|c|c|c|c|c|c|}
\hline \multirow{2}{*}{ Variable } & \multicolumn{5}{|c|}{ MetS Score } & \multirow{2}{*}{ F Value } & \multirow{2}{*}{ P Value } \\
\hline & 0 & 1 & 2 & 3 & 4 & & \\
\hline \multicolumn{8}{|c|}{ ATP } \\
\hline Insulin (pg/ml) & $45.04 \pm 23.55$ & $13.97 \pm 5.62$ & $127.40 \pm 58.10$ & $50.91 \pm 25.30$ & $39.54 \pm 13.46$ & $\mathrm{~F}_{4,120}=1.4600$ & 0.2258 \\
\hline Leptin (ng/ml) & $15.72 \pm 7.44$ & $13.90 \pm 5.70$ & $24.46 \pm 11.09$ & $25.02 \pm 13.46$ & $35.11 \pm 16.35$ & $\mathrm{~F}_{4,120}=0.6965$ & 0.5973 \\
\hline Leptin/Adiponectin Ratio & $0.01 \pm 0.00$ & $0.01 \pm 0.00$ & $0.02 \pm 0.01$ & $0.02 \pm 0.00$ & $0.02 \pm 0.00$ & $\mathrm{~F}_{4,120}=0.3536$ & 0.8405 \\
\hline \multicolumn{8}{|c|}{ IDF } \\
\hline Insulin (pg/ml) & & $33.54 \pm 12.93$ & $97.74 \pm 68.60$ & $117.00 \pm 58.30$ & $55.43 \pm 24.67$ & $\mathrm{~F}_{3,122}=1.499$ & 0.2238 \\
\hline Adiponectin (ng/ml) & & $1.29 \pm 0.12$ & $1.49 \pm 0.11$ & $1.38 \pm 0.11$ & $2.10 \pm 0.10$ & $\mathrm{~F}_{3,122}=1.0350$ & 0.3835 \\
\hline Leptin (ng/ml) & & $12.77 \pm 6.43$ & $23.64 \pm 12.21$ & $25.45 \pm 13.22$ & $25.65 \pm 10.29$ & $\mathrm{~F}_{3,122}=0.5073$ & 0.6787 \\
\hline Leptin/Adiponectin Ratio & & $0.01 \pm 0.00$ & $0.02 \pm 0.00$ & $0.02 \pm 0.00$ & $0.01 \pm 0.00$ & $\mathrm{~F}_{3,122}=1.8060$ & 0.1558 \\
\hline \multicolumn{8}{|c|}{ WHO } \\
\hline Insulin (pg/ml) & & $33.44 \pm 19.77$ & $79.10 \pm 38.00$ & $121.50 \pm 52.65$ & $52.54 \pm 24.45$ & $\mathrm{~F}_{3,122}=1.8010$ & 0.1564 \\
\hline Adiponectin (ng/ml) & & $1.28 \pm 0.12$ & $1.66 \pm 0.11$ & $1.30 \pm 0.15$ & $2.26 \pm 0.12$ & $\mathrm{~F}_{3,122}=2.2360$ & 0.0433 \\
\hline Leptin (ng/ml) & & $12.22 \pm 9.21$ & $24.30 \pm 15.55$ & $23.49 \pm 12.38$ & $30.45 \pm 13.36$ & $\mathrm{~F}_{3,122}=0.6795$ & 0.5680 \\
\hline Leptin/Adiponectin Ratio & & $0.01 \pm 0.00$ & $0.01 \pm 0.00$ & $0.02 \pm 0.01$ & $0.01 \pm 0.00$ & $\mathrm{~F}_{3,122}=0.9963$ & 0.4007 \\
\hline
\end{tabular}


Table 4. Insulin, Adiponectin levels stratified by Presence or Absence of MetS components.

\begin{tabular}{ccccccc}
\hline \multirow{2}{*}{ Variable } & \multicolumn{3}{c}{ Insulin (pg/ml) } & \multicolumn{3}{c}{ Adiponectin (ng/ml) } \\
\cline { 2 - 7 } & Absent & Present & P Value & Absent & Present & P Value \\
\hline \multicolumn{5}{c}{ ATP III } \\
\hline $\begin{array}{c}\text { Abdominal } \\
\text { Obesity-WC }\end{array}$ & $98.51 \pm 44.60$ & $12.33 \pm 4.98$ & 0.0704 & $1.46 \pm 0.12$ & $1.71 \pm 0.11$ & 0.4868 \\
Raised FBG & $29.44 \pm 13.80$ & $104.63 \pm 76.39$ & 0.0389 & $1.93 \pm 0.10$ & $1.94 \pm 0.12$ & 0.1243 \\
Raised TG & $87.88 \pm 26.53$ & $40.37 \pm 10.33$ & 0.5982 & $1.51 \pm 0.13$ & $1.59 \pm 0.11$ & 0.8579 \\
Raised BP & $92.87 \pm 34.76$ & $82.62 \pm 54.48$ & 0.8859 & $1.74 \pm 0.11$ & $1.49 \pm 0.11$ & 0.6419 \\
Reduced HDL-c & $106.20 \pm 87.49$ & $43.66 \pm 12.54$ & 0.0372 & $1.27 \pm 0.10$ & $2.49 \pm 0.13$ & 0.0002 \\
\hline & & IDF & & & & \\
\hline Abdominal & $49.44 \pm 2.32$ & $98.46 \pm 16.99$ & 0.1583 & $1.47 \pm 0.11$ & $1.54 \pm 0.11$ & 0.8285 \\
Obesity-WC & & & & & \\
Raised FBG & $42.63 \pm 12.80$ & $106.45 \pm 79.74$ & 0.0383 & $1.92 \pm 0.10$ & $1.39 \pm 0.11$ & 0.1243 \\
Raised TG & $87.59 \pm 33.48$ & $40.54 \pm 27.80$ & 0.4822 & $1.57 \pm 0.11$ & $1.59 \pm 0.11$ & 0.8579 \\
Raised BP & $70.41 \pm 45.47$ & $84.27 \pm 48.44$ & 0.3685 & $1.81 \pm 0.11$ & $1.44 \pm 0.11$ & 0.3272 \\
Reduced HDL-c & $97.88 \pm 38.36$ & $33.34 \pm 14.32$ & 0.0937 & $1.28 \pm 0.11$ & $2.22 \pm 0.09$ & 0.0025 \\
\hline & & WHO & & & \\
\hline $\begin{array}{c}\text { Central } \\
\text { Obesity-WHR }\end{array}$ & $50.47 \pm 21.35$ & $86.44 \pm 53.71$ & 0.3897 & $1.53 \pm 0.12$ & $1.52 \pm 0.11$ & 0.9908 \\
Raised FBG & $42.33 \pm 28.76$ & $97.48 \pm 48.74$ & 0.0083 & $1.91 \pm 0.10$ & $1.40 \pm 0.11$ & 0.1243 \\
Dyslipidemia & $106.36 \pm 72.47$ & $19.56 \pm 11.28$ & 0.0341 & $1.25 \pm 0.11$ & $2.22 \pm 0.07$ & 0.0011 \\
Raised BP & $43.54 \pm 13.37$ & $92.44 \pm 37.26$ & 0.1074 & $1.65 \pm 0.11$ & $1.47 \pm 0.11$ & 0.5364 \\
\hline
\end{tabular}

Table 5. Leptin, Leptin/Adiponectin ratio stratified by presence or absence of MetS components.

\begin{tabular}{|c|c|c|c|c|c|c|}
\hline \multirow{2}{*}{ Variable } & \multicolumn{3}{|c|}{ Leptin (ng/ml) } & \multicolumn{3}{|c|}{ Leptin/Adiponectin Ratio } \\
\hline & Absent & Present & $P$ value & Absent & Present & Pvalue \\
\hline \multicolumn{7}{|c|}{ ATP } \\
\hline Abdominal Obesity-WC & $22.46 \pm 12.32$ & $29.24 \pm 11.34$ & 0.0946 & $0.02 \pm 0.00$ & $0.02 \pm 0.00$ & 0.6348 \\
\hline Raised FBG & $22.12 \pm 210.32$ & $24.21 \pm 11.33$ & 0.5433 & $0.01 \pm 0.00$ & $0.01 \pm 0.00$ & 0.1845 \\
\hline Raised TG & $21.32 \pm 10.65$ & $46.44 \pm 22.24$ & 0.0023 & $0.01 \pm 0.00$ & $0.03 \pm 0.01$ & 0.2593 \\
\hline Raised BP & $17.38 \pm 9.21$ & $23.37 \pm 12.43$ & 0.4133 & $0.01 \pm 0.00$ & $0.02 \pm 0.00$ & 0.4962 \\
\hline Reduced HDL-c & $22.39 \pm 12.54$ & $24.54 \pm 10.48$ & 0.2481 & $0.02 \pm 0.00$ & $0.01 \pm 0.00$ & 0.0017 \\
\hline \multicolumn{7}{|c|}{ IDF } \\
\hline Abdominal Obesity-WC & $23.58 \pm 11.37$ & $24.99 \pm 12.71$ & 0.6392 & $0.02 \pm 0.00$ & $0.02 \pm 0.00$ & 0.2845 \\
\hline Raised TG & $20.26 \pm 11.27$ & $44.27 \pm 24.92$ & 0.0019 & $0.01 \pm 0.00$ & $0.03 \pm 0.01$ & 0.4329 \\
\hline Raised BP & $23.52 \pm 11.39$ & $24.37 \pm 14.88$ & 0.4819 & $0.01 \pm 0.00$ & $0.02 \pm 0.00$ & 0.6299 \\
\hline Reduced HDL-c & $23.44 \pm 12.63$ & $23.98 \pm 12.59$ & 0.7372 & $0.02 \pm 0.00$ & $0.01 \pm 0.00$ & 0.1893 \\
\hline \multicolumn{7}{|c|}{ WHO } \\
\hline Central Obesity-WHR & $21.49 \pm 10.61$ & $25.37 \pm 12.88$ & 0.4922 & $0.01 \pm 0.00$ & $0.02 \pm 0.00$ & 0.6391 \\
\hline Raised FBG & $22.68 \pm 11.41$ & $24.51 \pm 12.49$ & 0.2718 & $0.01 \pm 0.00$ & $0.02 \pm 0.00$ & 0.0928 \\
\hline Dyslipidemia & $23.27 \pm 15.66$ & $25.61 \pm 12.55$ & 0.6449 & $0.02 \pm 0.01$ & $0.01 \pm 0.00$ & 0.0426 \\
\hline Raised BP & $21.16 \pm 11.54$ & $26.43 \pm 15.49$ & 0.2844 & $0.01 \pm 0.00$ & $0.02 \pm 0.00$ & 0.6291 \\
\hline
\end{tabular}


Diabetic participants with the presence of reduced HDL-Cholesterol levels showed significantly lower levels of insulin for NCEP-ATP III criteria (43.66 \pm 12.54 against $106.20 \pm 87.49)$, significantly higher adiponectin levels for the NCEP-ATP III $(2.49 \pm 0.13$ against $1.27 \pm 0.10)$ and IDF criteria $(2.22 \pm 0.09$ against $1.28 \pm 0.11)$ and significantly lower leptin/adiponectin ratio (ATP) (0.01 \pm 0.00 against $0.02 \pm 0.00$ in comparison with diabetic participants without presence of reduced HDL-Cholesterol levels. Participants with the presence of Dyslipidaemia which includes reduced HDL levels in its definition (WHO criteria) showed significantly lower levels of insulin (19.56 \pm 11.28 against $106.36 \pm$ 72.47), significantly higher levels of adiponectin (2.22 \pm 0.07 against $1.25 \pm 0.11)$ and a significantly lower leptin/adiponectin ratio $(0.01 \pm 0.00$ against $0.02 \pm$ 0.01) in comparison to participants without the presence of Dyslipidaemia.

When the diabetic participants were stratified based on the presence or absence of SD domains (Table 6), participants who had impotence showed significantly lower leptin/adiponectin ratio $(0.01 \pm 0.00$ against $0.02 \pm 0.01)$ but no significant difference in the levels of insulin, adiponectin, leptin whilst participants with the presence of premature ejaculation showed no difference in insulin, adiponectin, leptin and leptin/adiponectin ratios in comparison with subjects without the presence of premature ejaculation. Participants with the presence of non-sensuality showed significantly higher values of leptin /adiponectin ratio $(0.03 \pm 0.00$ against $0.02 \pm 0.00)$ but did not show any significant difference in the levels of insulin, adiponectin and leptin when compared to participants

Table 6. Sexual Dysfunction (SD), SD subscales against hormonal assays.

\begin{tabular}{|c|c|c|c|c|c|c|}
\hline \multirow{2}{*}{ Variable } & \multicolumn{3}{|c|}{ Insulin (pg/ml) } & \multicolumn{3}{|c|}{ Adiponectin (ng/ml) } \\
\hline & Absent & Present & P Value & Absent & Present & P Value \\
\hline Impotence & $106.42 \pm 79.32$ & $51.37 \pm 22.43$ & 0.1103 & $1.31 \pm 0.12$ & $1.70 \pm 0.10$ & 0.1522 \\
\hline Premature ejaculation & $50.32 \pm 22.40$ & $119.00 \pm 55.10$ & 0.0682 & $1.39 \pm 0.11$ & $1.64 \pm 0.10$ & 0.3828 \\
\hline Non sensuality & $124.68 \pm 89.76$ & $66.44 \pm 34.36$ & 0.1135 & $1.36 \pm 0.13$ & $1.57 \pm 0.26$ & 0.5087 \\
\hline Avoidance & $91.63 \pm 51.82$ & $73.72 \pm 43.94$ & 0.4201 & $1.66 \pm 0.18$ & $1.35 \pm 0.13$ & 0.2666 \\
\hline Dissatisfaction & $46.30 \pm 3.54$ & $113.40 \pm 98.40$ & 0.0265 & $1.38 \pm 0.15$ & $1.60 \pm 0.10$ & 0.4384 \\
\hline Non communication & $54.39 \pm 17.40$ & $101.48 \pm 62.44$ & 0.0419 & $2.06 \pm 0.18$ & $1.37 \pm 0.10$ & 0.0438 \\
\hline \multirow[t]{3}{*}{ Infrequency } & $39.33 \pm 18.55$ & $100.98 \pm 72.20$ & 0.1310 & $2.15 \pm 0.11$ & $1.28 \pm 0.10$ & 0.0042 \\
\hline & \multicolumn{3}{|c|}{ Leptin (ng/ml) } & \multicolumn{3}{|c|}{ Leptin/Adiponectin ratio } \\
\hline & Absent & Present & P Value & Absent & Present & P Value \\
\hline Impotence & $24.20 \pm 12.24$ & $22.29 \pm 11.63$ & 0.4879 & $0.02 \pm 0.01$ & $0.01 \pm 0.00$ & 0.0184 \\
\hline Premature ejaculation & $22.83 \pm 12.48$ & $25.46 \pm 12.22$ & 0.2590 & $0.02 \pm 0.00$ & $0.02 \pm 0.01$ & 0.6293 \\
\hline Non sensuality & $25.32 \pm 11.46$ & $24.64 \pm 12.22$ & 0.3174 & $0.02 \pm 0.00$ & $0.03 \pm 0.00$ & 0.0103 \\
\hline Avoidance & $28.39 \pm 18.43$ & $20.75 \pm 13.66$ & 0.4573 & $0.02 \pm 0.00$ & $0.02 \pm 0.00$ & 0.5492 \\
\hline Dissatisfaction & $25.37 \pm 12.38$ & $23127 \pm 12.99$ & 0.3753 & $0.02 \pm 0.00$ & $0.01 \pm 0.00$ & 0.1093 \\
\hline Non communication & $22.28 \pm 12.44$ & $25.98 \pm 12.72$ & 0.9862 & $0.01 \pm 0.00$ & $0.02 \pm 0.00$ & 0.2603 \\
\hline Infrequency & $23.24 \pm 11.39$ & $25.53 \pm 12.77$ & 0.3854 & $0.01 \pm 0.00$ & $0.02 \pm 0.00$ & 0.0431 \\
\hline
\end{tabular}


without the presence of non-sensuality. There were no significant differences recorded in the levels of adiponectin, leptin and leptin/adiponectin ratio levels for diabetic male participants with or without the presence of Dissatisfaction problems. However a significantly higher levels of insulin was recorded for participants with the presence of Dissatisfaction problems (113.40 \pm 98.40 against $46.30 \pm 3.54$ ) in comparison to those without dissatisfaction problems. Participants with the presence of Non communication problems showed significantly higher levels of insulin (101.48 \pm 62.44 against $54.39 \pm 17.40)$ and lower levels of adiponectin (1.37 \pm 0.10 against $2.06 \pm 0.18)$ whilst participants who experienced infrequency showed significantly lower levels of adiponectin and a higher leptin/adiponectin ratio in comparison with participants without the presence of infrequency problems ( $1.28 \pm 0.10$ against $2.15 \pm 0.11$ and $0.01 \pm 00$ against $0.02 \pm 0.00)$.

When the $-\mathrm{SD} /-\mathrm{MetS},+\mathrm{SD} /-\mathrm{MetS},-\mathrm{SD} /+\mathrm{MetS}$ and $+\mathrm{SD} /+$ MetS groups were compared for insulin, adiponectin, leptin and leptin/adiponectin ratio, there was a significant difference in the insulin levels for NCEP-ATP III ( $\mathrm{p}=0.0186, \mathrm{~F}_{3,122}$ $=3.5722), \operatorname{IDF}\left(\mathrm{p}=0.0487, \mathrm{~F}_{3,122}=2.6982\right)$ and adiponectin levels for NCEPATP III criteria $\left(\mathrm{p}=0.0256, \mathrm{~F}_{3,122}=1.0633\right)$, WHO criteria $\left(\mathrm{p}=0.0396, \mathrm{~F}_{3,122}=\right.$ 2.5945). Insulin levels was generally highest among the $+\mathrm{SD} /-\mathrm{MetS}$ group, followed by the $+\mathrm{SD} /+\mathrm{MetS}$ group, with the $-\mathrm{SD} /+\mathrm{MetS}$ group generally recording the lowest insulin levels. For adiponectin, the lowest levels were generally recorded in the $-\mathrm{SD} /-\mathrm{MetS}$ with the $+\mathrm{SD} /+\mathrm{MetS}$ group generally recorded the highest levels of adiponectin. When the assayed hormonal parameters were considered as risk factors for SD and the MetS after adjustment for age and BMI, none of the assayed hormonal parameters recorded as a determinant of either the MetS or SD.

\section{Discussion}

Subjects with reduced HDL-cholesterol levels recorded higher levels of adiponectin whilst Dyslipidaemia which has reduced HDL-cholesterol levels in its definition also recorded significantly higher levels of adiponectin. This could explain the potential role of adiponectin levels in the pathogenesis of the MetS and possibly diabetes. This is further supported by the observation of higher levels of leptin (ATP/IDF) amongst subjects with raised triglyceride levels which is a cardinal event in the early metabolic derangements involved in the pathogenesis of the MetS. Since raised triglycerides is a marker of increased fatty acid availability, it is possible that higher leptin levels could have triggered the suppression of testosterone levels via leptin receptors present on leydig cells or by the increased aromatization of androgens to oestrogens. This could potentially cause a reduction in the levels of free and bioavailable testosterone in circulation thus inducing a state of hypogonadism. Whether the increased leptin levels in a state of increased abdominal obesity (Table 4) directly results in the reduction in testosterone levels via suppression of production mediated by leptin receptors on leydig cells or by the increased aromatization to oestrogens, reduced testosterone levels could without doubt further promote adipocytosis as decreased testosterone le- 
vels has been shown to promote adipocytosis in various research. The resultant increase in adipocytes coupled with the hypogonadism could be a major trigger in the events that leads to development of the MetS. Furthermore, as testosterone levels decrease further in the face of persistent increase in adipocytes, Nitric Oxide (NO) availability could be compromised by direct inactivation of eNOS. A reduction in the availability of NO in the endothelial wall could result in increased free radical damage and increased oxidative stress which will eventually compromise endothelial integrity. As NO availability is compromised in the presence of a progressive and persistently unresolved dyslipidaemic derangements and hyperglycaemia, neuronal glycation coupled with unavailability of NO could trigger the series of events which eventually promotes the progression from the MetS characterised by dyslipidaemia to a competition for the remaining NO. Thus a vicious cycle could be created between endothelial dysfunction, the MetS and SD. The importance of the fine balance between the levels of adiponectin and leptin is showed by the lower leptin/adiponectin ratio recorded for participants with reduced HDL levels, Dyslipidaemia (Table 4), Impotence and Non-sensuality (Table 5). It is therefore not surprising that the participants with the presence of Impotence (ED) which is the SD sub component likely to be directly affected by NO unavailability recorded significantly lower values for the leptin/adiponectin ratio.

The finding of lower levels of adiponectin in participants with problems of Non-communication and Infrequency in sexual activity could be due to the important role of adiponectin as an anti-inflammatory hormone. It is possible that as endothelial dysfunction progresses, with attendant increase in inflammatory mediators, the decreasing levels of adiponectin could have triggered a state of increased oxidative stress that could have resulted in tiredness and a general lack of energy and interest to engage in sexual activity thus reflecting as sexual infrequency problems. This is supported by the observation of a higher leptin/adiponectin ratio seen among participants with problems of infrequency in sexual activity. Thus the role of pro and anti-inflammatory mediators in endothelial dysfunction could be equally important in the mechanisms that results in sexual dysfunction. It is therefore not surprising that various researchers have identified endothelial dysfunction as a common denominator underlining both MetS and SD. This is also supported by the observation in this study that insulin and adiponectin levels showed significant differences when various shades of MetS/ SD were compared (Table 7). But the fact that leptin did not show a difference when the various shades of MetS/SD were compared could mean that increased leptin levels could possibly have been an earlier event before the development of both MetS and SD. Thus leptin could have played a significant role in the development of diabetes and thus could not have been varied among a group of diabetics with various shades of MetS/SD but still remained an important mediator as evidenced by the significance in the ratio of leptin to adiponectin seen in this study. Even though none of the assayed hormonal parameters recorded as determinants of SD and the MetS (Table 8), a longitudinal study which meas- 
ures a range of both pro and anti-inflammatory hormones and adipokines as risk factors for SD and the MetS could potentially aid in assessing the risk of developing these conditions among diabetics.

Table 7. Hormonal parameters stratified by the various shades of the SD/MetS groups.

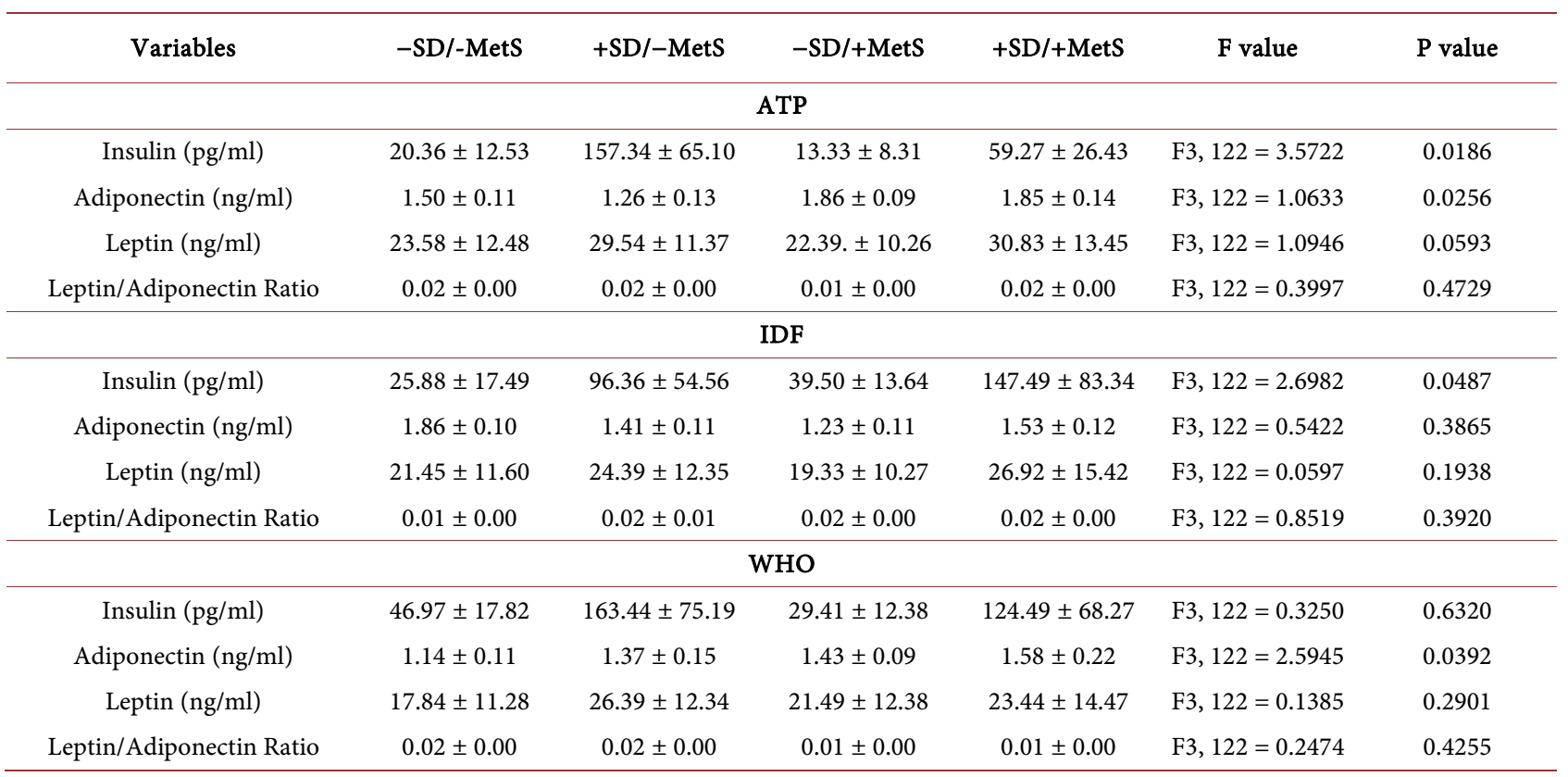

Table 8. Hormonal Determinants of Sexual Dysfunction and MetS.

\begin{tabular}{|c|c|c|c|c|}
\hline Variable & OR (CI) & P Value & $\mathrm{aOR}(\mathrm{CI})$ & P Value \\
\hline \multicolumn{5}{|l|}{ SEXUAL } \\
\hline \multicolumn{5}{|c|}{ DYSFUNCTION } \\
\hline \multicolumn{5}{|c|}{ Insulin } \\
\hline \multicolumn{5}{|l|}{ Normal } \\
\hline High & $0.00(0.00-0.00)$ & 1.0000 & $0.00(0.00-0.00)$ & 1.0000 \\
\hline \multicolumn{5}{|l|}{ Leptin } \\
\hline \multicolumn{5}{|l|}{ Normal } \\
\hline High & $0.91(0.08-10.60)$ & 0.9390 & $0.62(0.04-8.87)$ & 0.7260 \\
\hline \multicolumn{5}{|c|}{ Adiponectin } \\
\hline \multicolumn{5}{|l|}{ Normal } \\
\hline High & $0.86(0.06-9.46)$ & 0.8549 & $0.54(0.02-7.65)$ & 0.7044 \\
\hline \multicolumn{5}{|c|}{ METABOLIC } \\
\hline \multicolumn{5}{|c|}{ SYNDROME } \\
\hline \multicolumn{5}{|l|}{ Insulin } \\
\hline \multicolumn{5}{|l|}{ Normal } \\
\hline Low & $8.43(2.06-10.43)$ & 0.9880 & $5.83(1.04-8.76)$ & 0.9800 \\
\hline \multicolumn{5}{|l|}{ Leptin } \\
\hline \multicolumn{5}{|l|}{ Normal } \\
\hline High & $0.22(0.02-2.59)$ & 0.2290 & $0.26(0.02-3.75)$ & 0.3250 \\
\hline \multicolumn{5}{|c|}{ Adiponectin } \\
\hline \multicolumn{5}{|l|}{ Normal } \\
\hline High & $0.75(0.08-7.66)$ & 0.6949 & $0.43(0.01-6.35)$ & 0.5430 \\
\hline
\end{tabular}




\section{Conclusion}

The relative balance between pro inflammatory and anti-inflammatory mediators of endothelial dysfunction could possibly be essential for the progression of diabetes to SD and the MetS thus a longitudinal study which measures a range of both pro and anti-inflammatory hormones and adipokines as risk factors for SD and the MetS could potentially aid in improvements in assessing the risk of developing these conditions among diabetics.

\section{Competing Interests}

The authors declare that they have no competing interests.

\section{Authors' Contributions}

WKBAO, NA, HA, CO and CKGS developed the concept and designed the study. NA, WKBAO, HA, PPMD, CO and ATB administered the questionnaire, analysed and interpreted the data. NA, WKBAO, HA, PPMD, CO and ATB performed all the assay procedures. WKBAO, HA, NA, CO, PPMD and ATB drafted the manuscript. NA, HA, WKBAO, PPMD, CKGS and ATB revised the manuscript for intellectual content. All authors read and approved the final manuscript.

\section{Ethics, Consent and Permissions}

Ethical approval was obtained from the Committee on Human Research Publication and Ethics (CHRPE) of the School of Medical Science and the KomfoAnokye Teaching Hospital (KATH), Kumasi. All participants gave an informed and signed consent to partake in this study.

\section{Consent for Publication}

All participants gave an informed and signed consent for research to be used for publication.

\section{References}

[1] Wild, S., Roglic, G., Green, A., Sicree, R. and King, H. (2004) Global Prevalence of Diabetes Estimates for the Year 2000 and Projections for 2030. Diabetes Care, 27, 1047-1053. https://doi.org/10.2337/diacare.27.5.1047

[2] Feldman, H.A., Goldstein, I., Hatzichristou, D.G., Krane, R.J. and McKinlay, J.B. (1994) Impotence and Its Medical and Psychosocial Correlates: Results of the Massachusetts Male Aging Study. Journal of Urology, 151, 54-61.

[3] Braun, M., Wassmer, G., Klotz, T., Reifenrath, B., Mathers, M. and Engelmann, U. (2000) Epidemiology of Erectile Dysfunction: Results of the Cologne Male Survey. International Journal of Impotence Research, 12, 305-311. https://doi.org/10.1038/sj.ijir.3900622

[4] Moreira, E.D., Lbo, C.F.L., Diament, A., Nicolosi, A. and Glasser, D.B. (2003) Incidence of Erectile Dysfunction in Men 40 to 69 Years Old: Results from a Population-Based Cohort Study in Brazil. Urology, 61, 431-436.

[5] Wang, C., Jackson, G., Jones, T.H., Matsumoto, A.M., Nehra, A., Perelman, M.A., 
Swerdloff, R.S., Traish, A., Zitzmann, M. and Cunningham, G. (2011) Low Testosterone Associated with Obesity and the Metabolic Syndrome Contributes to Sexual Dysfunction and Cardiovascular Disease Risk in Men with Type 2 Diabetes. Diabetes Care, 34, 1669-1675. https://doi.org/10.2337/dc10-2339

[6] Jackson, G., Betteridge, J., Dean, J., Eardley, I., Hall, R., Holdright, D., Holmes, S., Kirby, M., Riley, A. and Sever, P. (2002) A Systematic Approach to Erectile Dysfunction in the Cardiovascular Patient: A Consensus Statement-Update 2002. International Journal of Clinical Practice, 56, 663-671.

[7] Laumann, E.O., Nicolosi, A., Glasser, D.B., Paik, A., Gingell, C., Moreira, E. and Wang, T. (2005) Sexual Problems among Women and Men Aged 40-80 y: Prevalence and Correlates Identified in the Global Study of Sexual Attitudes and Behaviors. International Journal of Impotence Research, 17, 39-57.

https://doi.org/10.1038/sj.ijir.3901250

[8] Kalter-Leibovici, O., Wainstein, J., Ziv, A., Harman-Bohem, I., Murad, H. and Raz, I. (2005) Clinical, Socioeconomic, and Lifestyle Parameters Associated with Erectile Dysfunction among Diabetic Men. Diabetes Care, 28, 1739-1744. https://doi.org/10.2337/diacare.28.7.1739

[9] Esposito, K., Giugliano, F., Martedì, E., Feola, G., Marfella, R., D’Armiento, M. and Giugliano, D. (2005) High Proportions of Erectile Dysfunction in Men with the Metabolic Syndrome. Diabetes Care, 28, 1201-1203. https://doi.org/10.2337/diacare.28.5.1201

[10] Brownlee, M. (2001) Biochemistry and Molecular Cell Biology of Diabetic Complications. Nature, 414, 813-820. https://doi.org/10.1038/414813a

[11] Alexander, C.M., Landsman, P.B., Teutsch, S.M. and Haffner, S.M. (2003) NCEPDefined Metabolic Syndrome, Diabetes, and Prevalence of Coronary Heart Disease among NHANES III Participants Age 50 Years and Older. Diabetes, 52, 1210-1214. https://doi.org/10.2337/diabetes.52.5.1210

[12] Shaw, D., Hall, W. and Williams, C. (2005) Metabolic Syndrome: What Is It and What Are the Implications? Proceedings of the Nutrition Society, 64, 349-357. https://doi.org/10.1079/PNS2005442

[13] Haffner, S.M., Shaten, J., Stem, M.P., Smith, G.D., Kuller, L. and Group, M.R. (1996) Low Levels of Sex Hormone-Binding Globulin and Testosterone Predict the Development of Non-Insulin-Dependent Diabetes Mellitus in Men. American Journal of Epidemiology, 143, 889-897.

https://doi.org/10.1093/oxfordjournals.aje.a008832

[14] Kaplan, S.A., Meehan, A.G. and Shah, A. (2006) The Age Related Decrease in Testosterone Is Significantly Exacerbated in Obese Men with the Metabolic Syndrome. What Are the Implications for the Relatively High Incidence of Erectile Dysfunction Observed in These Men? The Journal of Urology, 176, 1524-1528.

[15] Bays, H., Mandarino, L. and Defronzo, R.A. (2004) Role of the Adipocyte, Free Fatty Acids, and Ectopic Fat in Pathogenesis of Type 2 Diabetes Mellitus: Peroxisomal Proliferator-Activated Receptor Agonists Provide a Rational Therapeutic Approach. The Journal of Clinical Endocrinology \& Metabolism, 89, 463-478. https://doi.org/10.1210/jc.2003-030723

[16] McTernan, P.G., McTernan, C.L., Chetty, R., Jenner, K., Fisher, F.M., Lauer, M.N., Crocker, J., Barnett, A.H. and Kumar, S. (2002) Increased Resist in Gene and Protein Expression in Human Abdominal Adipose Tissue. The Journal of Clinical Endocrinology \& Metabolism, 87, 2407. https://doi.org/10.1210/jcem.87.5.8627

[17] Selvin, E., Feinleib, M., Zhang, L., Rohrmann, S., Rifai, N., Nelson, W.G., Dobs, A., Basaria, S., Golden, S.H. and Platz, E.A. (2007) Androgens and Diabetes in 
Men: Results from the Third National Health and Nutrition Examination Survey (NHANES III). Diabetes Care, 30, 234-238. https://doi.org/10.2337/dc06-1579

[18] Wang, C., Cunningham, G., Dobs, A., Iranmanesh, A., Matsumoto, A.M., Snyder, P.J., Weber, T., Berman, N., Hull, L. and Swerdloff, R.S. (2004) Long-Term Testosterone Gel (AndroGel) Treatment Maintains Beneficial Effects on Sexual Function and Mood, Lean and Fat Mass, and Bone Mineral Density in Hypogonadal Men. The Journal of Clinical Endocrinology \& Metabolism, 89, 2085-2098.

https://doi.org/10.1210/jc.2003-032006

\section{List of Abbreviations}

\begin{tabular}{cl}
\hline ADT & Androgen Deprivation Therapy \\
AV & Avoidance \\
BP & Blood Pressure \\
DIS & Dissatisfaction \\
NO & Nitric Oxide \\
ED & Erectile Dysfunction \\
GRISS-M & Golombuk-Rust Inventory for Sexual Satisfaction-Male \\
IMP & Impotence \\
INF & Infrequency \\
IDF & International Diabetes Federation \\
MetS & Metabolic syndrome \\
NCEP-ATP III & National Cholesterol Education Programme-Adult Treatment Panel III \\
NO & Nitric Oxide \\
NS & Non-sensuality \\
PE & Premature Ejaculation \\
SHBG & Sex Hormone Binding Globulin \\
SD & Sexual Dysfunction \\
\hline
\end{tabular}


Submit or recommend next manuscript to SCIRP and we will provide best service for you:

Accepting pre-submission inquiries through Email, Facebook, LinkedIn, Twitter, etc. A wide selection of journals (inclusive of 9 subjects, more than 200 journals)

Providing 24-hour high-quality service

User-friendly online submission system

Fair and swift peer-review system

Efficient typesetting and proofreading procedure

Display of the result of downloads and visits, as well as the number of cited articles Maximum dissemination of your research work

Submit your manuscript at: http://papersubmission.scirp.org/

Or contact asm@scirp.org 